\title{
An Intelligent System for Structural Analysis-Based Design Improvements
}

\author{
M. Novak, B. Dolšak
}

The goal of the research work presented in this paper was to collect, organize, and write the knowledge and experience about structural analysis-based design improvements into a knowledge base for a consultative advisory intelligent decision support system. The prototype of the system presented proposes possible design changes that should be taken into consideration to improve the design candidate according to the results of a prior stress-strain or thermal analysis. The system can be applied either in the design of new products or as an educational tool.

Keywords: computer-aided design, structural optimisation, knowledge based systems, decision support

\section{Introduction}

Engineering design is a very complex, iterative process. Physical and mathematical modelling simulations and analyses are computationally intensive but offer immense insight into a developing product. Structural engineering analysis plays an important role in this process, as the results of such analysis are often used as basic optimization parameters to improve the design candidate being validated and analysed. The number of iterations/cycles that are needed to reach the final design solution depends directly on the quality of the initial design and the appropriateness of the subsequent design changes.

A wide range of Computer Aided Design (CAD) software is extensively applied in performing various design activities, such as modelling, kinematics, simulations, structural analysis or just drawing technical documentation. Nowadays, CAD software can be so complex, and offers such an extensive assortment of different options, that one can easily be confused. This is the reason for the relatively low level of control over these systems. In many cases, CAD software is used just as a "black box". This often leads to completely wrong conclusions, especially when young engineers facing complex design problems do not understand the basic theories, or their knowledge and experience are simply too limited.

Mainly because of the problems mentioned above, many professional engineers involved in the product development process share the opinion that the benefits of applying CAD are below expectations. It cannot be denied that modern CAD tools provide a wide range of technical support for the designers. However, these tools are unable to provide adequate help in more creative parts of the design process involving complex reasoning [1], as for example, when a design candidate needs to be evaluated and further design decisions need to be made. Thus, many design steps, including the analysis-based design improvement process, still depend mostly on the designer's knowledge and experience.

The goal of our research work presented here was to increase the intelligence of existing CAD tools [2] by collecting, organizing, and encoding this kind of knowledge and experience into a knowledge base for the consultative advisory intelligent decision support system. The prototype of the system proposes possible design changes that should be taken into consideration to improve the design candidate according to the results of prior stress-strain or thermal analysis [3]. The system is called PROPOSE, and can be applied either in the process of designing new products or for education purposes.

\section{Analysis-based design improvement}

Every proposed design should be verified during the embodiment phase of the design process. The purpose of engineering analysis in the design process is to simulate and verify the conditions in the structure, as they will appear during its exploitation. If the structure does not satisfy the given criteria, it needs to be improved by applying certain design optimisation steps.

Several redesign steps are usually possible for design improvement. The selection of one or more redesign steps to be performed in a certain case depends on the user's requirements, possibilities and wishes. The easiest design change is to select a different material. However, in many cases, this cannot be done or cannot be financially justified. Fig. 1 presents some basic ways to improve analysis-based design. If the structure is over-dimensioned, it is "on the safe side", because it is stronger than the loading requires. However, if such a design solution is too heavy or too expensive, redesign is still justified. On the other hand, design changes are necessary for under-dimensioned structure, which cannot bear the loadings and will fail during its exploitation.

Nowadays, the results of structural analyses are usually very well-presented. The analysing software is very helpful at this point, as it offers adequate computer-graphic support in terms of reasonably clear pictures showing the distribution of the computed values for unknown parameters, such as stresses, deformations and temperatures inside the body of the structure [4]. These values are then compared with the allowable limits, defined either by the selected material (stresses, temperatures) or by specific design requirements (deformations).

However, the support provided by geometry-based CAD systems is limited, because of the wide semantic gap between the expressive power of the geometry and the abstract features of the product. Thus, substantial knowledge and experience is needed in order to understand the results of the analysis and to choose appropriate redesign actions [5]. The 


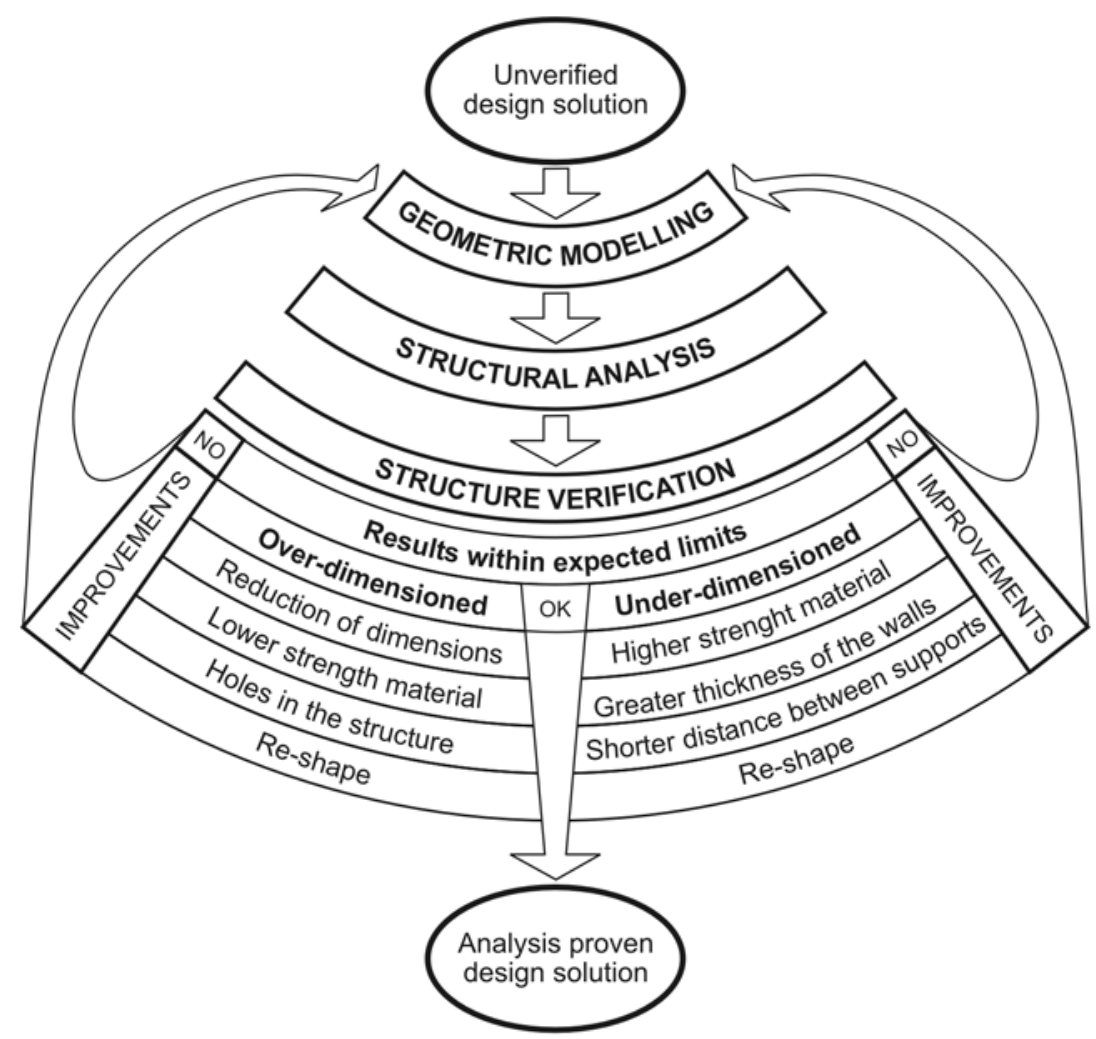

Fig. 1: Some basic steps in the analysis-based design improvement process

experience gained by many design iterations are of crucial importance.

\section{Intelligent design improvement process}

Many important characteristics of advanced computing applications are changing the way engineers interact with computers. New approaches based on Artificial Intelligence (AI) have earned acceptance in many fields of engineering and have started to emerge in commercial software. Analysis-based design improvement is certainly an engineering task, with a great potential for the application of intelligent systems.

Finite Element Analysis (FEA) is one of the most extensively used numerical methods in the engineering product development process [6]. Knowledge Based Engineering (KBE) techniques have been applied to FEA for over twenty years to teach [7], advise [8], automate the FEA pre-processing phase mainly involving automatic mesh generation [9], and verify calculations [10]. However, the use of AI methods is almost absent in the post-processing phase and the consequent design modification/improvement of designs [11, 12]. Many early examples present a rule-based approach to automate the optimisation of simple components or geometric shapes [13]. Recently, optimisation procedures have become a part of KBE systems for specific products [14, 15].

It is evident that KBE applications for analysis-based design improvement are quite scarce $[11,16]$, although the need for linking intelligent programs to structural analysis in design has been discussed in many research works, and some more specific AI techniques, like machine learning, had also proved to be serviceable in this particular domain [17]. In the last decade, research in this field has been concerned mainly with the integration of various software systems in such a way that the whole design process, including analysis, can be automated, again mostly for specific products [18, 19].

Various software and hardware components are frequently required to perform both geometric modelling and engineering analysis. In this context, an independent intelligent advisory system for decision support within the analysis-based design improvement process can be applied more easily. Moreover, using a qualitative description of engineering analysis results, such a system can be more general and cover a wider range of application areas. Intelligent interpretation of analysis results can be used to choose the most suitable design modifications [20]. Thus, what is needed is a mechanism for extracting meaningful qualitative design information from simulation results and to couple this information to a design modification system as a higher level of representation [21].

\section{PROPOSE - a consultative advisory intelligent system}

In order to provide intelligent decision support for a designer when performing analysis-based design improvement, we have developed a consultative intelligent system proposing appropriate modifications to design parameters. Development of the system has been carried out in a sequence of steps. Knowledge acquisition and development of the knowledge base were the first and most important. Theoretical and practical knowledge about design and possible design improvements were investigated and collected. After 


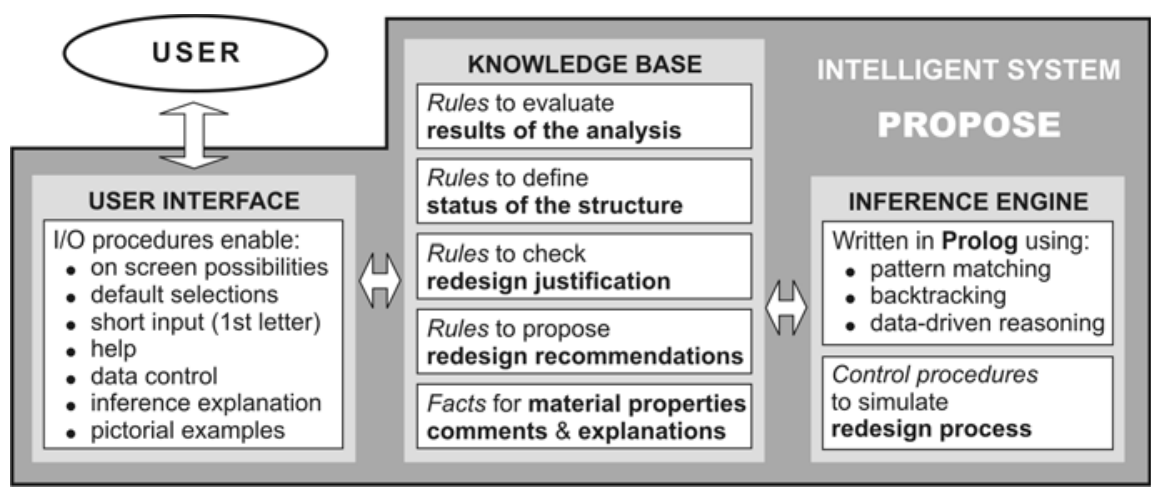

Fig. 2: Basic architecture of the PROPOSE system

that, appropriate representation formalism for the acquired knowledge was defined and the knowledge base of the system was encoded. Finally, we developed the shell of the system, named PROPOSE, consisting of the user interface and inference engine suited to the existing knowledge base (Fig. 2). The knowledge base and the shell of the system were encoded in Prolog syntax. Visual Prolog version 5.2, developed by Prolog Development Center A/S. (2001), was used for this purpose.

\subsection{Knowledge base of the system}

In the knowledge acquisition process we took advantage of all possible ways to acquire knowledge about design improvements, from a literature survey, including an examination of previously-conducted engineering analyses, to interviews with selected human experts. This was not a straight forward task. For example, many analysis reports contain confidential data and are thus unavailable for inclusion. Additionally, interviews and examination of existing redesign examples are conditioned by the quality of cooperation with the available experts, and can be time-consuming. The scope of such results is greatly limited by these considerations.

Production rules were chosen as the most appropriate knowledge representation formalism, because they have a well-defined form, which is transparent, modular and easy to understand. Furthermore, the form of the actual rules used by human experts in the design process is quite similar to the form of production rules. Each rule presents a list of recommended design changes that should be taken into consideration while dealing with a certain problem, subject to certain limits. The rules are generalised and do not refer exclusively to the examples that were used during the knowledge acquisition process. They can also be used for any new problem and its limits which match those at the head of the rule. In such a case, application of the appropriate rule would result in a list of recommended design changes for dealing with the given problem. The designer, with his or her own knowledge and experience, should chose and apply one or more design changes that are possible, reasonable, and maximally effective for each particular case. Some pictorial examples have been added to the system as an additional help to the user, to enhance understanding of the proposed design changes and to assist in making a suitable decision.

The present version of the PROPOSE knowledge base comprises 314 various types of rules and facts (Fig. 2) that are necessary for the system to be functional. For example, several rules are needed just to define the status of the structure (not stiff enough, under-dimensioned, over-dimensioned or satisfactory). The status of the structure depends on the type of engineering analysis, the parameters being analysed and the deviations between computed and allowable values. Finally, the need for redesign is defined considering the status of the structure, the scale of the proposed changes (significant or minor) and justification for redesign.

From technical point of view, the most important rules in the knowledge base are those defining redesign recommendations. Let us present an example of such a rule for advising a designer how to reduce the local stress gradients around a hole in a plate, which is a quite frequent structural engineering design problem. Fig. 3 shows a tensile loaded "infinite" plate with a circular hole (a) and three different

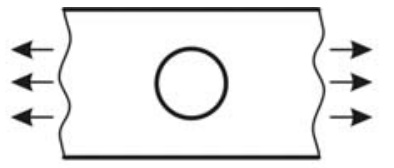

(a)

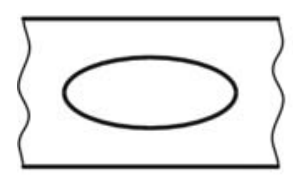

(b)

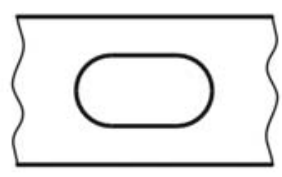

(c)

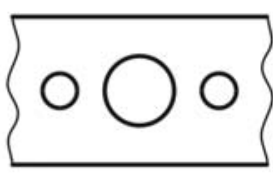

(d)

Fig. 3: Reducing local stresses around a circular hole in a tensile loaded "infinite" plate 
design improvement options for reducing the potential high stresses around the hole (b, c, d).

By generalising the geometric appearance of the hole, the following rule for design improvement recommendations can be defined:

\section{IF the stresses are too high \\ AND structure is a 3D "infinite" plate \\ AND the critical area is around the hole \\ THEN}

reduce the size of the hole

make a chamfer on the edge of the hole

change the circular hole into an elliptical hole (Fig. 3b)

change the circular hole into a round ended slot (Fig. 3c)

add smaller relief holes in the line of loads on both sides of the hole (Fig. 3d)

change the hole geometry to decrease the stress concentration factor $(\mathrm{K})$

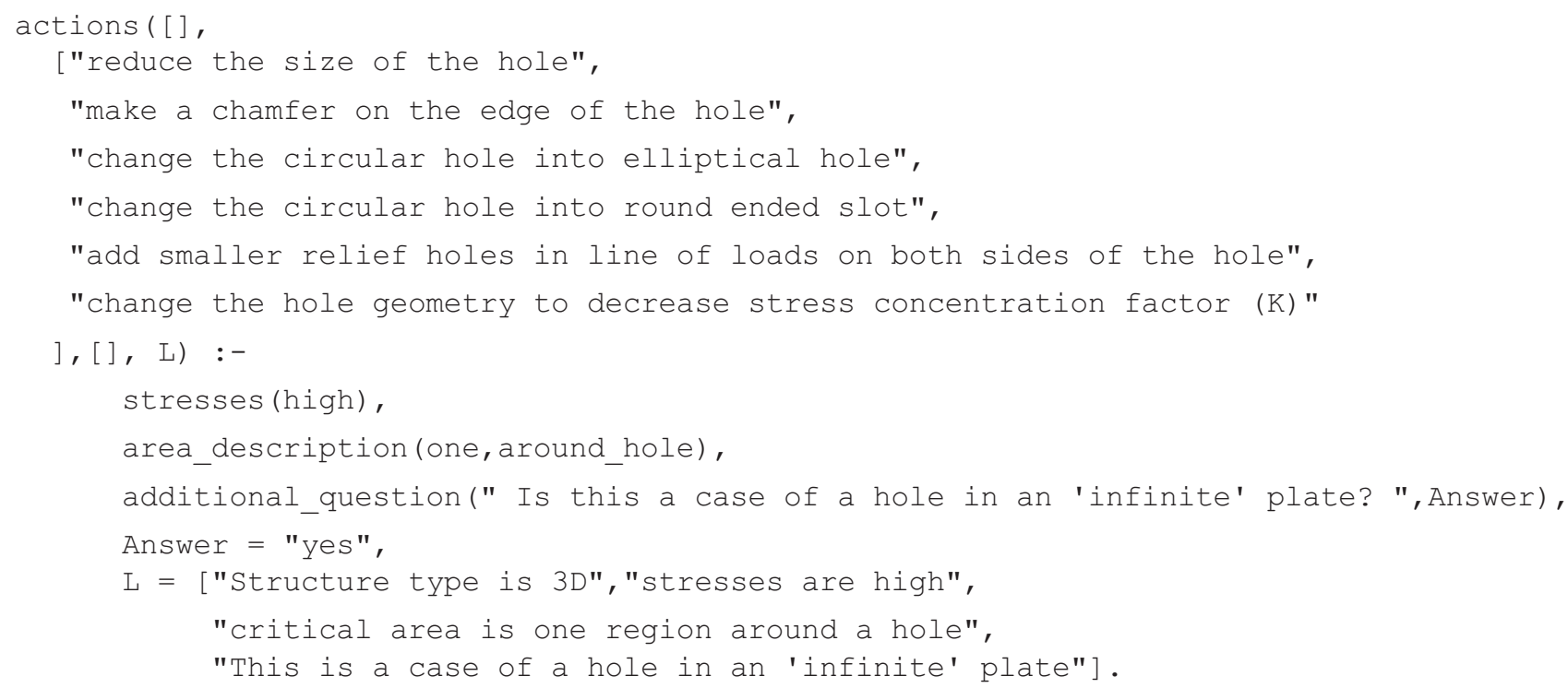

\subsection{Shell of the system}

The shell of the PROPOSE system was encoded in Prolog. Prolog was chosen because of its built-in features such as rule-based programming, pattern matching and backtracking, which are excellent tools for developing intelligent systems [22]. Our work concentrated on a declarative presentation of the knowledge, using data-driven reasoning, which is built into Prolog. However, some control procedures were also added to the inference engine of the system to adjust the performance to the real-life design process.

For the user interface, our goal was to simulate communication between the designer-beginner and the designer-expert. The user interface enables the user to input the data, informs the user about the results, offers help and presents information about the inference process. As can be seen in Fig. 2 , the user interface has many features including help. Thus enables efficient and user friendly communication, although the system is run as a simple monolithic console application. Due to the simple architecture of the system, the response times are very modest (within a few seconds). It is however evident that PROPOSE is a prototype which is still the subject of research and, as such, cannot be compared with commercial software.

\section{Application of the PROPOSE system}

Application of the PROPOSE system is based on interactive communication between the user and the system aiming to define the status of a structure and to generate the list of redesign proposals, if applicable (Fig. 4).

It is reasonable to use the PROPOSE system when the results of the analysis are available and also reliable. The system offers some basic guidelines to help the user to clarify whether, for example, FEA results are reliable and can serve as basic parameters for verifying the suitability of the design. However, validation and determination of the reliability of the FEA results should form part of the analysis. Therefore, the aim of the system presented here is only to emphasize the importance of the reliability of the results and to present some guidelines, as a kind of help when considering the reliability of the results.

After the availability and reliability of the analysis results have been confirmed, the user has to present the type of analysis that has been performed prior to the PROPOSE application. The current version of the system can deal with 


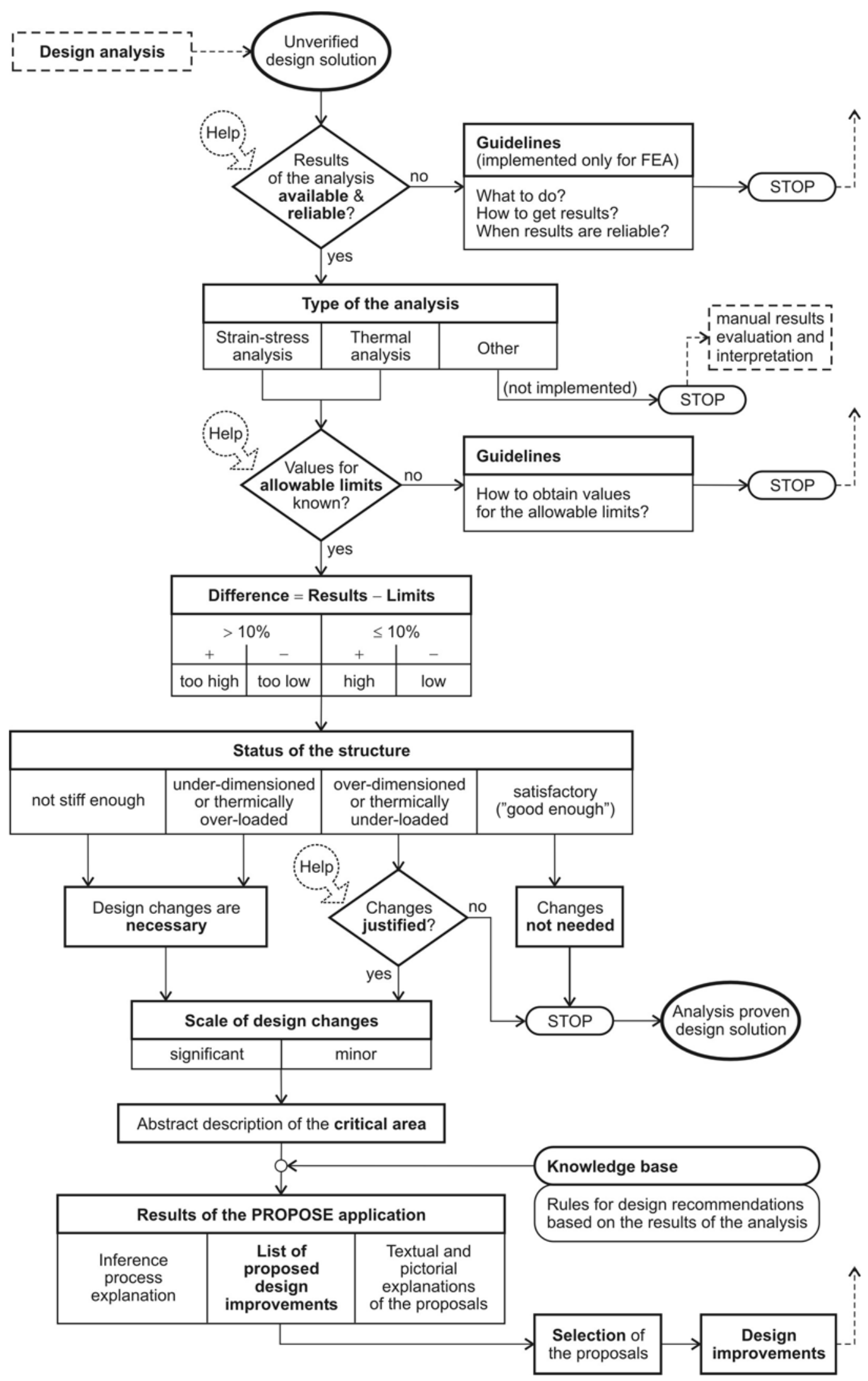

Fig. 4: Diagram representation of the PROPOSE application

strain-stress or thermal analyses. In the next step, the user needs to know the allowable values for the structure being analysed. A qualitative description needs to be defined of the deviation between the computed maximum values for the stresses, deformations or temperatures and the allowable lim- its. Considering the range of differences between the actual values and the allowable limits, the system defines the status of the design candidate (satisfactory, not stiff enough, under or over dimensioned), and what kind of changes need to be made (significant, minor or none). 


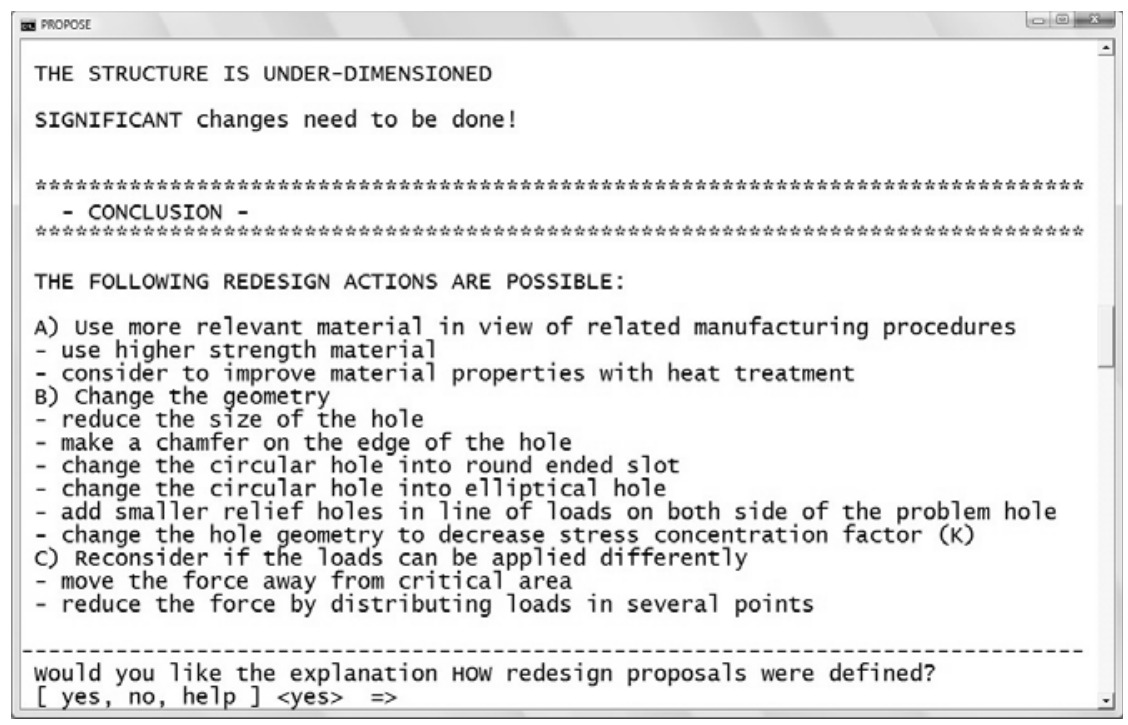

Fig. 5: The result of a PROPOSE application

In cases where design changes are advised, any improvement of the design candidate should be justified. If the structure is not stiff enough or if it is under-dimensioned, design changes are necessary and the system itself classifies them as justified. On the other hand, if the structure is over-dimensioned, the user/designer has to decide whether design changes are justified or not. As in many other cases, the user can obtain some help from the system when making this decision.

In order to present some recommendations for design improvement, the critical area where the computed values exceed the allowable limits needs to be defined. For the time being, the system can deal with two types of structure: beams and general three-dimensional structures. An abstract description of the critical area is supported by a list of predefined features, e.g., around a hole, a notch, in the corner. A critical area should be defined as generally as possible, to cover the majority of problems that may occur in practice. Presently, the number of predefined geometric features is relatively small. However, by answering some additional questions, a critical area can be described in a more detailed manner.

For each problem described, the system searches for redesign proposals in the knowledge base and presents them on the screen. As can be seen in Fig. 5, the system is able to provide three types of proposals, the first referring to material changes, the second to geometry changes, and the last type of proposals referring to loads.

As already mentioned, the user can obtain an insight into the inference process or obtain more information about certain redesign proposals. An example of a pictorial explanation for a redesign recommendation is presented in Fig. 6.

\section{Conclusions}

The PROPOSE system is a knowledge-based module to be applied within the computer-aided design optimisation cycle to increase the intelligence of existing CAD tools in order to enable more intelligent and less experience-dependent design performance [23].

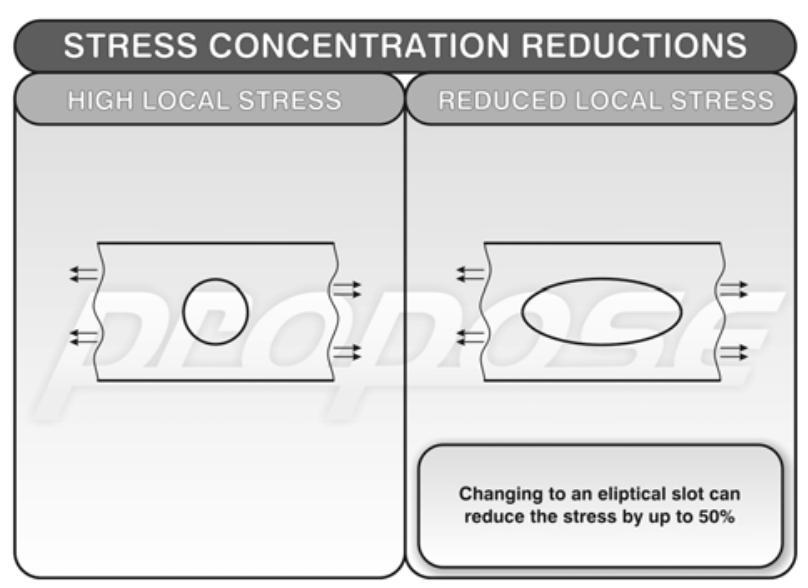

Fig. 6: Pictorial explanation of a redesign proposal

The research on analysis-based design improvement dealt mostly with the pre-processing phase of the analysis, while much less attention was paid to the post-processing phase, which is well known as the other bottleneck in the analysis process. In order to fill in this gap, a prototype of the intelligent advisory system was developed to assist a designer in the post-processing phase of structural analysis by proposing possible design changes that should be taken into consideration to improve the design according to the results of a prior analysis.

The PROPOSE system offers help and advice on how to solve design problems in abstractly described critical areas of the structure after a stress-strain or thermal analysis. The architecture of the system, based on production rules, enables the system to be expanded relatively easily with additional rules, for example for a more specific description of any problem, for other types of engineering analyses, and for a deeper, multi-physics understanding of recommended design changes.

When using the PROPOSE system, a designer has to answer some questions stated by the system to present the results of the engineering analysis qualitatively, with emphasis on the 
critical area that needs to be optimised. These answers are then compared with the rules in the knowledge base, and the most appropriate design changes that should be taken into account for the various cases are determined and recommended to the user. The system provides constant support for the user's decisions in terms of explanations and advice.

The PROPOSE system is not intended only for optimising new products in practice, but also for use in design education. In fact, the system is currently used in the design education process at the Faculty of Mechanical Engineering, University of Maribor, Slovenia. Overall experience with the operation of the system has been very positive and encouraging. The important feature of the system, the ability to explain the inference process, is especially welcome for students, as it enables them not only to select appropriate further design steps, but also to acquire some new knowledge.

\section{References}

[1] Mili, F. et al.: Knowledge Modelling for Design Decisions. Artificial Intelligence in Engineering, Vol. 15 (2001), p. 153-164.

[2] McMahon, C., Browne, J.: CADCAM: Principles, Practice and Manufacturing Management. Prentice Hall, $2^{\text {nd }}$ edition, 1999.

[3] Dolšak, B., Novak, M., Jezernik, A.: Intelligent Design Optimisation based on the Results of Finite Element Analysis. The International Journal of Advanced Manufacturing Technology, Vol. 21 (2003), p. 391-396.

[4] Tichánek, R. et al.: Structural Stress Analysis of an Engine Cylinder Head. Acta Polytechnica, Vol. 45 (2005), No. 3, p. 43-48.

[5] Ong, Y. S., Keane, A. J.: A Domain Knowledge Based Search Advisor for Design Problem Solving Environments. Engineering Applications of Artificial Intelligence, Vol. 15 (2002), p. 105-116.

[6] Zienkiewicz, O. C. et al.: The Finite Element Method: its Basis and Fundamentals. Oxford: Elsevier Butterwoth-Heinemann, $6^{\text {th }}$ edition, 2005.

[7] Forde, B., Stiemer, S.: ESA: Expert structural analysis for engineers. Computers and Structures, Vol. 29 (1988), p. 171-174.

[8] Turkiyyah, G. M., Fenves, S. J.: Knowledge-Based Assistance for Finite-Element Modelling. Intelligent Systems, Vol. 11(1996), No. 3, p. 23-32.

[9] Dolšak, B.: Finite Element Mesh Design Expert System. Knowledge-Based Systems, Vol. 15 (2002), p. 315-322.

[10] Breitfeld, T., Kroplin, B.: An Expert System for the Verification of Finite-Element Calculations. In: Proc. $4^{\text {th }}$ Int. Symposium on Assessment of Software Tools (SAST'96), IEEE Computer Society, 1996, p. 18-23.

[11] Smith, L., Midha, P.: A Knowledge-based System for Optimum and Concurrent Design and Manufacture by Powder Metallurgy Technology. Int. Journal of Prod. Res., Vol. 7 (1999), No. 1, p. 125-137.

[12] Burczynski, T. et al.: Optimization and Defect Identification Using Distributed Evolutionary Algorithms. Engi- neering Applications of Artificial Intelligence, Vol, 17 (2004), p. 337-344.

[13] Jozwiak, S.: Artificial Intelligence: The Means for Developing More Effective Structural Optimisation Programs. In: Proc. Int. Conf. on Comp. Mechanics (ICCM 86), 1986, p. 103-108.

[14] Chapman, C. B., Pinfold, M.: The Application of a Knowledge Based Engineering Approach to the Rapid Design and Analysis of an Automotive Structure. Advances in Engineering Software, Vol. 32 (2001), p. 903-912.

[15] Ríos, J. et al.: KBE Application for the Design and Manufacture of HSM Fixtures. Acta Polytechnica, Vol. 45 (2005), No. 3, p. 17-24.

[16] Pilani, R. et al.: A Hybrid Intelligent Systems Approach for Die Design in Sheet Metal Forming. International Journal of Advanced Manufacturing Technology, Vol. 16 (2000), p. 370-375.

[17] Dolšak, B., Bratko, I., Jezernik, A.: Knowledge Base for Finite Element Mesh Design Learned by Inductive Logic Programming. Artificial Intelligence for Engineering Design, Analysis and Manufacturing, Vol. 12 (1998), p. 95-106.

[18] Custom Product Engineering During the Sales Cycle. Pittsburgh, USA: ANSYS User Group Conference, 2000.

[19] Kowalski, Z. et al.: An Expert System for Computer Aided Design of Ship Systems Automation. Expert Systems with Applications, Vol. 20 (2001), p. 261-266.

[20] Armstrong, C. and Bradle, B.: Design Optimisation by Incremental Modification of Model Topology. In: Proc. $8^{\text {th }}$ Int. Meshing Roundtable, Sandia National Laboratories, 1999, p. 293-298.

[21] Sahu, K., Grosse, I.: Concurrent Iterative Design and the Integration of Finite Element Analysis Results. Engineering with Computers, Vol. 10 (1994), p. 245-257.

[22] Bratko, I.: Prolog: programming for artificial intelligence. Addison-Wesley, $3^{\text {rd }}$ edition, 2000.

[23] Dolšak, B., Novak, M., Kaljun, J.: Intelligent Support for a Computer Aided Design Optimization Cycle. Acta Polytechnica, Vol. 46 (2006), No. 5, p. 15-20.

Marina Novak, Ph.D.

phone: +38622207692

e-mail:marina.novak@uni-mb.si

Bojan Dolšak, Ph.D.

phone: +38622207691

Fax: +38622207994

e-mail:dolsak@uni-mb.si

Laboratory for Intelligent CAD systems

University of Maribor

Faculty of Mechanical Engineering

Smetanova 17

SI-2000 Maribor, Slovenia

http://licads.fs.uni-mb.si/ 